\title{
Invited commentary on ... When unbearable suffering incites psychiatric patients to request euthanasia: a qualitative study ${ }^{\dagger}$
}

Steve Pearce

\author{
Summary \\ Although the paper in this issue by verhofstadt et al \\ sheds some light on the features of unbearable suffering \\ stemming from psychiatric disorder leading to a request \\ for euthanasia, this is limited. The bulk of the paper \\ illustrates the particular ethical challenges posed by \\ the policy of making euthanasia available in these \\ circumstances.
}

\section{Declaration of interest}

S.P. is the chair of Oxford Health NHS Trust Clinical Ethics Advisory Group, and vice chair of the Royal College of Psychiatrists Professional Practice and Ethics Committee.

\section{Copyright and usage}

(c) The Royal College of Psychiatrists 2017.
Verhofstadt et $a l^{1}$ present data from patients attending a private psychiatrist (one of the authors) with a view to obtaining agreement to undergoing euthanasia for suffering as a result of one or more psychiatric conditions. In so doing they have attempted to contribute to a definition of unbearable suffering that could be used in future to decide on the outcome of such requests in the Low Countries, where it forms part of the legal criteria. The paper expands knowledge of what unbearable suffering in such a context might look like.

Some countries that permit euthanasia (lethal treatment administered by a doctor) or assisted suicide (death brought about by the actions of the patient but assisted by a professional or lay person) permit it only in the context of terminal illness. Belgium, Luxembourg and The Netherlands permit euthanasia in those who are neither suffering from a terminal illness, nor suffering severe and untreatable physical pain. In Holland this group of patients makes up only $3 \%$ of requests (mostly suffering from affective disorder), of which only $2 \%$ are approved. ${ }^{2}$ It is this group of patients, in whom a request for euthanasia stems from reported psychological suffering, with which this paper is concerned.

\section{The challenges of defining unbearable psychological suffering}

The extent to which the paper is able to expand a clinically useful definition of unbearable psychological suffering, as opposed to illustrating possible causes and associations of psychological suffering, is limited. The authors note that often unbearable suffering is very long term, sometimes extending back to an early age; there is often a gradual worsening of the experience; it can be accompanied by the perception that there is no prospect of improvement; and that the experience exceeds the patient's capacity to cope. These features are either tautological (an experience exceeding the patient's capacity to cope) or shared with bearable suffering. The authors note that the extent to which suffering is unbearable can only be determined 'from the perspective of the patient themselves, and may depend on their physical and mental strength and personality'. It may be that a reliable way of deciding whether psychological distress is unbearable is not achievable, leaving doctors relying on the strength of the patient's desire to escape their situation.

${ }^{\dagger}$ See pp. 238-245 and 248-249, this issue.
The larger part of the paper deals with the reported causes or associations of psychological suffering that has led to the patient seeking euthanasia. The authors note that unbearable suffering is 'highly affected by psychological, socioenvironmental, existential and biographical factors'. The impact of socioeconomic factors such as poverty, lack of appropriate resources in the health or social care system, social isolation, and difficulties communicating with loved ones and professionals, illustrate the multifactorial nature of psychological suffering, and undermines an ethical argument that euthanasia is an appropriate or proportionate response. The contribution of burdensomeness to some patient's pessimism recorded in the paper is particularly concerning. If poverty is contributing to psychological suffering, or resource limitations are restricting the interventions available to alleviate it, the appropriate response is to address these issues rather than recommend euthanasia for a patient affected by them. To offer euthanasia in such a context is to attempt to put right a systemic or societal inequality or lack by removing the person suffering the effects of it.

The paper also illustrates the difficulties encountered when trying to separate out the effects of mental disorder from the 'rational desires' of sufferers. ${ }^{3}$ Mental disorders affect complex functions such as motivation, social processes and cognition, which are likely to have an impact on a person's assessment of their future. Some disorders have specific effects that are problematic in this regard. Depressive disorder produces cognitive distortions about the future, manifesting in pessimism and hopelessness. A patient with resistant depression, for whom all currently available strategies have been exhausted, may have good reason to feel pessimistic, but differentiating this from pessimism arising as a symptom of the illness may not be possible, either for the patient or for the clinician (see Brandt ${ }^{4}$ for further discussion of this point). The point here is that pessimism, for example about whether the patient is unlikely ever to be reconciled to a child, may be misplaced if it is a symptom of the psychiatric disorder. Similarly, borderline personality disorder produces periods of sincerely felt suicidality, often accompanied by reports of lifelong unhappiness and dissatisfaction with life, interspersed with periods of optimism. This pattern might reasonably be regarded as fulfilling criteria for unbearable suffering if a longitudinal view is not available, for example if the patient's psychiatrist is not involved in the application. In this sample the diagnosis was made in $15 \%$, with a further $30 \%$ suffering from other personality disorders including personality disorders not otherwise specified, 
which often shares features with borderline personality disorder. It may be possible to address such difficulties with legislation such as is in force in Switzerland, where assisted suicide is allowed in patients with psychiatric disorder only with a psychiatric report declaring that the wish for suicide is not part of the mental disorder but is 'self-determined, well considered, and constant. ${ }^{5}$ Controversies around suicides accompanied by written statements of intent in patients with personality disorder illustrate the difficulties that can arise in making such nuanced longitudinal judgements. ${ }^{6}$

\section{The issue of uncertainty in psychiatric diagnosis}

The decision to agree to euthanasia in the patients presented in the paper is founded at least in part on diagnosis. There is a degree of uncertainty in psychiatric diagnosis that is unusual in the rest of medicine. ${ }^{7}$ Psychiatric diagnostic categories represent syndromes containing significant heterogeneity rather than disease entities with established pathophysiological causes, ${ }^{8}$ and diagnostic stability is poor. ${ }^{9}$ For example, it is not uncommon for someone judged to have depression unresponsive to treatment later to be thought to have bipolar disorder, leading to a change of treatment that may prove more effective. Such diagnostic uncertainty arising in a patient with cancer would be likely to prevent referral for assisted dying or euthanasia in those jurisdictions in which it is allowed, although diagnostic clarity is not required for euthanasia in Belgium or The Netherlands. In many psychiatric conditions, interventions that have been tried in the past may prove useful at another time, and changing levels of social support can have an impact on recovery. ${ }^{10,11}$ The scenario in which a patient with pancreatic cancer suffering unbearable pain can be said to be untreatable is different to the way someone with bipolar disorder or borderline personality disorder might be thought of as untreatable.

\section{Reluctance of psychiatrists to get involved in euthanasia}

Some of the patients in the cohort complained that the process of applying for euthanasia was drawn out. Verhofstadt et $a l^{1}$ wonder if more efficiency might alleviate some of their suffering by making the process less stressful. Patients presenting with a euthanasia request should be treated compassionately and advised accurately, but doctors must be able to decline to be involved. Making the process more transparent may address concerns that euthanasia procedures in Belgium have been insufficiently monitored. Although reporting of euthanasia cases is compulsory, a survey in 2010 found that only half of cases were reported, with reasons for not reporting including physicians not regarding their actions as euthanasia, worries that the legal process had not been observed, and the burden of paperwork. ${ }^{12}$ The difficulties treating suffering stemming from psychiatric disorder as grounds for euthanasia may be one reason for reports in the paper that several patients had difficulty finding physicians who were willing to take part in the process of referral for the procedure. Most physicians approached by this cohort appear to have been reluctant to participate, or in some cases even to enter into discussions. Psychiatrists have been found to be more reluctant than other physicians to participate in euthanasia or assisted dying, ${ }^{2}$ in the cases referred to by Verhofstadt et al this may relate to doubts about the reliability of judgements of capacity and the meaning of symptoms in severe intractable psychiatric disorder.

Most countries regard suicide in individuals with mental illness as an undesirable outcome, to be addressed by improved practice, more or better targeted resources, and appropriate population-level interventions. ${ }^{13}$ The idea of psychiatric euthanasia poses a challenge to this approach, proposing that there is a group of patients who should either be allowed to kill themselves, aided in doing so, or killed by physicians on the basis of the level and intractability of their psychiatric symptoms, even in the absence of high-quality services.

\section{Families and euthanasia}

Finally, the paper paints an optimistic picture of the involvement of relatives. Complaints from relatives that they are excluded from participating in the mental healthcare of their loved ones on the basis of confidentiality ${ }^{14}$ are likely to be heightened in a health system that allows psychiatric euthanasia. There is some anecdotal evidence of this. ${ }^{15}$

\section{Conclusions}

In the absence of a terminal illness or chronic untreatable pain with established cause, euthanasia for severe suffering as a result of psychiatric disorder poses ethical risks that have not yet been adequately addressed. Many of these are illustrated in this paper. ${ }^{1}$ Mental health services are poorly funded compared with other health sectors, ${ }^{16}$ and it is more common for patients to feel unsupported, or to report being unable to access care on a frequent and timely basis, than it is in physical illness. Under these conditions, euthanasia might be the wrong answer to the correct question: why do many psychiatric patients feel so desperate.

Steve Pearce, MRCP, MRCPsych, Complex Needs Service, Oxford Health

NHS Foundation Trust Manzil Way, Oxford, OX4 1XE UK.

Email: steve.pearce@oxfordhealth.nhs.uk

First received 20 Mar 2017, accepted 14 Jul 2017

\section{References}

1 Verhofstadt M, Thienpont L, Peters G-JY. When unbearable suffering incites psychiatric patients to request euthanasia: qualitative study. $\mathrm{Br} J$ Psychiatry 2017; 211: 238-45.

2 Levy TB, Azar S, Huberfeld R, Siegel AM, Strous RD. Attitudes towards euthanasia and assisted suicide: a comparison between psychiatrists and other physicians. Bioethics 2013; 27: 402-8.

3 Savulescu J. Rational desires and the limitation of life-sustaining treatment. Bioethics 1994; 8: 191-222.

4 Brandt RB. The rationality of suicide. In Suicide: The Philosophical Issues (eds M Battin, DJ Mayo): 117-32. St Martin's Press, 1980.

5 Steck N, Egger M, Maessen M, Reisch T, Zwahlen M. Euthanasia and assisted suicide in selected European countries and US states: systematic literature review. Med Care 2013; 51: 938-44.

6 David AS, Hotopf M, Moran P, Owen G, Szmukler G, Richardson G. Mentally disordered or lacking capacity? Lessons for management of serious deliberate self harm. BMJ 2010; 341: c4489.

7 Frances A. The new crisis of confidence in psychiatric diagnosis. Ann Intern Med 2013; 159: 221-2.

8 Cuthbert BN. Research Domain Criteria: toward future psychiatric nosologies. Dialogues Clin Neurosci 2015; 17: 89-97.

9 Baca-Garcia E, Perez-Rodriguez MM, Basurte-Villamor I, Fernandez Del Moral $A L$, Jimenez-Arriero MA, Gonzalez De Rivera JL, et al. Diagnostic stability of psychiatric disorders in clinical practice. Br J Psychiatry 2007; 190: 210-6.

10 Platt J, Keyes KM, Koenen KC. Size of the social network versus quality of social support: which is more protective against PTSD? SOc Psychiatry Psychiatr Epidemiol 2014; 49: 1279-86.

11 Brown GW, Harris T. Social Origins of Depression: A Study of Psychiatric Disorder in Women. Routledge, 2012.

12 Smets T, Bilsen J, Cohen J, Rurup ML, Mortier F, Deliens L. Reporting of euthanasia in medical practice in Flanders, Belgium: cross sectional analysis of reported and unreported cases. BMJ 2010; 341: c5174.

13 Mann JJ, Apter A, Bertolote J, Beautrais A, Currier D, Haas A, et al. Suicide prevention strategies: a systematic review. JAMA 2005; 294: 2064-74.

14 Wilkinson $C$, McAndrew S. 'I'm not an outsider, I'm his mother!' A phenomenological enquiry into carer experiences of exclusion from acute psychiatric settings. Int J Ment Health Nurs 2008; 17: 392-401.

15 Aviv R. The death treatment. When should people with a non-terminal illness be helped to die? New Yorker 2015; 22 June.

16 Lancet Global Mental Health Group. Scale up services for mental disorders: a call for action. Lancet 2007; 370: 1241-52. 